\title{
Sociocultural dimension of epilepsy: An anthropological study among Guaraní communities in Bolivia-An International League Against Epilepsy/International Bureau for Epilepsy/World Health Organization Global Campaign Against Epilepsy Regional Project
}

\author{
Elisa Bruno ${ }^{\mathrm{a}}$, Alessandro Bartoloni ${ }^{\mathrm{b}}$, Vito Sofia ${ }^{\mathrm{a}}$, Florentina Rafael ${ }^{\mathrm{c}}$, Donata Magnelli ${ }^{\mathrm{b}}$, Elio Ortiz ${ }^{\mathrm{d}}$, \\ Sandra Padilla ${ }^{\mathrm{d}}$, Graziella Quattrocchi ${ }^{\mathrm{a}}$, Filippo Bartalesi ${ }^{\mathrm{b}}$, Higinio Segundo e, Mario Zappia a , \\ Pierre-Marie Preux ${ }^{c}$, Alessandra Nicoletti ${ }^{\mathrm{a}, *}$ \\ a Department GF Ingrassia, Section of Neurosciences, University of Catania, Catania, Italy \\ ${ }^{\mathrm{b}}$ Infectious and Tropical Diseases Unit, University of Florence, Florence, Italy \\ ${ }^{c}$ Institute of Neuroepidemiology and Tropical Neurology, EA 3174 Tropical and Comparative Neuroepidemiology, University of Limoges, Limoges, France \\ ' Centre for Anthropological Research of the Teko Guarani, Camiri, Bolivia \\ e Servicio Departamental de Salud, Santa Cruz, Camiri, Bolivia
}

\section{A R T I C L E I N F O}

\section{Article history:}

Received 23 May 2011

Revised 11 July 2011

Accepted 15 July 2011

Available online 15 August 2011

\section{Keywords}

Epilepsy

Ethnography

Cross-cultural

Medical anthropology

Traditional medicine

Global campaign against epilepsy

\begin{abstract}
A B S T R A C T
This study was performed to analyze sociocultural beliefs about epilepsy among Guaraní communities in Bolivia. People with epilepsy, their family members, the general population, and local health care personnel were interviewed about the meaning of and beliefs, feelings, and practices concerning epilepsy. Epilepsy is called mano-mano, a term that means being in a constant passage between life and death. The disease is attributed mainly to a failure to observe a fasting period and to other eating habits. Natural remedies are the most recommended treatments even though half of respondents reported that antiepileptic drugs may be effective. The concept of epilepsy as an embodied disease with natural causes appears to differ from that documented in other traditional societies. People with epilepsy do not represent a threat to the community, which seems to have an attitude aimed at their protection. Moreover, people from these communities appear to favor a combination of biomedical and traditional care systems.
\end{abstract}

(c) 2011 Elsevier Inc. All rights reserved.

\section{Introduction}

The epilepsy treatment gap, defined as the proportion of people with epilepsy (PWE) who require but do not receive appropriate treatment [1], varies worldwide, with a significantly higher peak in lower-income countries and rural areas [2,3]. In most of these settings it exceeds $75 \%$ for active epilepsy [4], reflecting a multidimensional nature linked to the lack of health resources, personnel, infrastructure, and treatment programs [5]. Moreover, the stigma associated with epilepsy and the cultural attitudes and beliefs of the local population often hinder adequate management and, thus, are considered additional factors that may contribute to the treatment gap [6-10].

A study conducted in the southeastern part of Bolivia (Cordillera province) among the Guaraní, a rural native society of South America, reported that only $10.5 \%$ of the identified PWE had ever taken

\footnotetext{
* Corresponding author at: Department GF Ingrassia, Section of Neurosciences, University of Catania, Via Santa Sofia 78-95123 Catania, Italy. Fax: + 390953782900. E-mail address: anicolet@unict.it (A. Nicoletti).
}

antiepileptic drugs (AEDs) longer than 2 months, resulting in a treatment gap near 90\% [11,12]. A 10-year follow-up study carried out on the same population in 2009 showed a substantially similar situation: $26.7 \%$ of PWE had taken AEDs for at least 2 months, but only $12.7 \%$ continued treatment for at least 1 year [13].

The Guaraní have a traditional magical and natural medical system as well as rites and traditions of ancestral origin. Knowledge is still orally transmitted and epilepsy is a well-recognized disease. Different surveys have reported that the cultural basis and preserved traditions of American societies influence their perception of diseases, social behavior, and maintenance of local treatments [14-17], affecting the relationship with public health services. The anthropological approach could represent an efficacious method to study this aspect and could also provide significant information to improve health strategies [18] and narrow the treatment gap at the community level. The latter point represents one of the main aims of the International League Against Epilepsy (ILAE)/International Bureau for Epilepsy (IBE)/World Health Organization (WHO) Global Campaign against Epilepsy (GCAE) Out of the Shadows initiative [19]. This study was performed as a part of the first phase of the recently approved GCAE 
Regional Project in Bolivia. The aim was to analyze sociocultural beliefs about epilepsy among the Guaraní, by exploring meaning attached to the disease and describing attitudes, traditional practices, and treatment strategies. The knowledge obtained might provide the basis for development of a biomedical program to treat epilepsy in this area.

\section{Methods}

\subsection{Study setting}

This work comprised anthropological fieldwork performed from January to March 2009 in the communities of Cañon de Segura and Rodeo in Bolivia (Latin America). The communities are located in the area of Camiri (Cordillera province), a city in the southwestern section of Santa Cruz Department in the area called the Gran Chaco. The study populations consisted of native Guaraní and descendants of intermarriage between Spanish colonists and the native tribes (mestizos). The Guaraní are one of the largest indigenous societies of Latin America. The country that is home to the largest group is Bolivia, with around 80,000 Guaraní living in the Gran Chaco area, according to the data of the Guaraní People Assembly (APG), an entity that represents more than 300 Guaraní communities of this country. Inhabitants in the rural areas near Camiri number about 3300 [20]. The community of Cañon de Segura has a population of 208, and the community of Rodeo, 322, according to the last census. Among the Guaraní, the first language is Guaraní, taught both informally within the communities and formally in public school. Currently, the language is still the main characteristic binding Guaraní society, even though most younger Guaraní are bilingual (speaking Spanish and Guaraní). In rural areas, the Guaraní live in poor dwellings and the local economy is based predominantly on agriculture and animal breeding. Cordillera province is administratively divided into 10 areas covering $86,245 \mathrm{~km}^{2}$, and the health care infrastructure in the province comprises a district hospital, nine area hospitals, and rural health centers situated in each community and managed by nurses and local health care personnel.

\subsection{Sample}

All the people included in the survey were Guaraní living in the communities at the time of the study. Community leaders and local health workers identified PWE living in the areas, their family members, and other relevant community members able to provide information about epilepsy and traditions (like the community's elders).

\subsection{Procedures}

The fieldwork was divided into two phases. As a first step, an ad hoc selected sample of the above-mentioned groups was invited to participate in focus group discussions (FGDs). The second phase of the work consisted of semistructured interviews of all responders identified in the communities as well as interviews of local health care personnel. The study was conducted with the agreement of the National Department of Epidemiology of the Ministry of Social Welfare and Public Health and with the support of the Guarani political organization (APG).

\subsubsection{Focus group}

Two focus groups consisting of 10 persons each were held. FGDs were conducted in the local language by health workers and personnel (Spanish/Guaraní bilingual) of the Centre for Anthropological Research of the Teko Guaraní, an institution aimed at restoring and enhancing Guaraní culture. The discussions took place at local health facilities. Anthropologists introduced topics for discussion focusing mainly on local terminology, the meaning attached to having epilepsy, orally transmitted beliefs, personal experiences, feelings toward PWE, and practices usually used by the communities to heal this disease. Each discussion was recorded. The aim of these FGDs was to to look at people's beliefs, attitudes, and opinions concerning the major topics during the first phase and then explore this information further during the second phase comprising the interviews.

\subsubsection{Interviews}

Semistructured interviews were conducted with PWE, their family members, the general Guaraní population, and community elders. The interviews were conducted in the native language and comprised 29 open-ended questions to encourage extended responses and explanations. Demographic characteristics were also recorded. Questions evolved from the FGDs covered presumed causes of epilepsy; actions and factors that could promote the disease; individual and community attitudes toward PWE such as prohibitions, forbidden activities, contacts, or places; traditional treatment options; knowledge of medical and social practices; identification of persons capable of dealing with the disease; places in which treatment should be carried out; and local perceptions regarding the biomedical approach (attitudes toward doctors, level of confidence). Separate interviews were conducted with local health care personnel focusing not only on their sociocultural vision of epilepsy, but also on the relationship between the communities and local health specialists and facilities.

\subsection{Data analysis}

Focus group discussions were recorded, and the transcripts were translated and reviewed by the research investigators. Qualitative analysis was performed by anthropologists who identified the main recurrent themes and subthemes, which were subsequently listed in tables and coded for retrieval and analysis. Quantitative data obtained by the interviews were processed using Stata Version 10 (Stata Corp., College Station, TX, USA) and Windows SPSS (Statistical Package for Social Sciences) Version 14.0 (SPSS Inc., Chicago, IL, USA). Results for quantitative variables are expressed as means, and those for qualitative variables, as frequencies and percentages.

\section{Results}

\subsection{Sample characteristics}

The questionnaire was administered to 47 people, 22 in Cañon de Segura and 25 in Rodeo. The study sample comprised 19 men and 28 women ( $\mathrm{M} / \mathrm{F}$ ratio: 0.68$)$, and the mean age was $41.4 \pm 15.0$ years (range: 15-86). Five respondents had epilepsy, 10 were family members of PWE, 7 were community elders, and 25 were other members of the community.

\subsection{Guaraní view on epilepsy}

\subsubsection{Meaning of the term}

Before investigating the origin, causes, and treatment of epilepsy in Guaraní communities, we focused on the local terminology used to refer to seizures and its meaning. Among the Guaraní, epilepsy is called mano-mano, which literally means "die-die" and refers to the concept of death with a notion of frequency (die several times) and also of being in a constant passage between life and death. In other terms, this word means always being on the border between life and death, reflecting the fact that mano-mano produces a constant interruption of life or a "partial death."

\subsubsection{Knowledge about epilepsy and its causes}

With respect to the nature and causes of epilepsy, the majority of respondents (45.0\%) indicated an unknown origin, $21.0 \%$ a natural origin, $15.0 \%$ a social origin, and $10.0 \%$ a supernatural origin. However, 
during the interview, it was noted that these responses were contradictory. In fact, even though $45.0 \%$ suggested the origin is unknown, $96.0 \%$ asserted that a practical action or a human behavior might cause the disease. Failure to observe the Yekuaku, a fasting period linked to special events, was the most often mentioned cause (Table 1). "It is caused by the nonrespect of the Yekuaku when someone dies or is born." Actually, according to Guaraní traditions, the Yekuaku must be followed by members of the community during special events that occur throughout their life, such as the transition from puberty to adulthood, death of a relative, and birth of a baby.

Eating habits also occupied an important place and were mentioned by $61.7 \%$ of responders, for example: "The disease is due to the consumption of forbidden food." Some food taboos were also associated with fasting. Thus, according to respondents, a person who ingests certain foods during this period could contract the disease: "It is a consequence of eating pork and goat meat during the Yekuaku."

Epilepsy was also attributed to alcohol consumption, social causes, and trauma. Furthermore, disease was also believed to be transmitted environmentally: "There is something in the environment that makes cows sick and also humans." Heredity represents a more marginal belief mentioned only by one person; supernatural causes also were seldom cited.

\subsubsection{Treatment of people with epilepsy}

More than half of respondents (57.4\%) stated knowing at least one treatment for epilepsy. In this group the elderly were more represented than young people aged less than 26 years. Natural remedies and special baths were the most recommended treatments, respectively, by 22.2 and $25.9 \%$ of respondents who commented: "The person should have a bath in the same place where pigs have a bath" (Table 2). Natural remedies often consisted of infusions: "The affected person should drink a tea made of curly feathers of a hen." It also appeared very important to take into account the time frame marked by natural phenomena such as the lunar cycle or days of the week: "For the treatment of epilepsy it should be considered the lunar cycle, especially the new moon and it is useful to soak the person with holy water during the attack"; "For recovering, they have to drink pig blood on a Friday with full moon." AEDs were mentioned only once.

Participants were asked about persons who can provide health care to PWE. With respect to the folk sector, the family, particularly the women in the family, was considered the best option to take care of PWE (Table 3). In considering which health specialists (among the

Table 1

Causes of epilepsy elicited from the Guaraní.

\begin{tabular}{lc}
\hline Answer & $N(\%)$ \\
\hline Practical actions & \\
Eating habits & $29(61.7)$ \\
Environmental agents & $3(6.4)$ \\
Parental neglect & $3(6.4)$ \\
Vision of spirits & $2(4.3)$ \\
Psychological trauma & $2(4.3)$ \\
Heredity & $1(2.1)$ \\
Alcohol ingestion & $1(2.1)$ \\
Detachment from the community & $0(0.0)$ \\
No answer & $6(12.8)$ \\
Total & $47(100.0)$ \\
Human behaviors & \\
Failure to observe the abstinence period (Yekuaku) & $30(63.8)$ \\
Detachment from own culture & $6(12.8)$ \\
Punishment for criticizing instead of helping & $4(8.5)$ \\
Underestimation of spiritual power & $2(4.3)$ \\
Curse & $2(4.3)$ \\
Violation of the rules of the community & $1(2.1)$ \\
Punishment for criticizing traditional medicine & $0(0.0)$ \\
No answer & $2(4.3)$ \\
Total & $47(100.0)$ \\
\hline
\end{tabular}

Table 2

Treatments for seizures known by 27 members of Guaraní communities.

\begin{tabular}{lc}
\hline Answer & $N(\%)$ \\
\hline Take a special bath & $7(25.9)$ \\
Natural cure & $6(22.2)$ \\
Blessing & $4(14.8)$ \\
Special diet & $3(11.1)$ \\
Take into account the lunar cycle & $2(7.4)$ \\
Take into account the days of the week & $2(7.4)$ \\
Prayers & $2(7.4)$ \\
Tablets & $1(3.8)$ \\
Total & $27(100)$ \\
\hline
\end{tabular}

traditional and professional sectors) could most likely provide care to PWE, $34.0 \%$ of respondents considered shamans' assistants (Poro-Peyu $\mathrm{Vae}$ ) to be the most capable of curing the disease. Community members also occupied an important place in this field, as $23.4 \%$ of respondents said that each community member who knows an effective treatment could be important in management of the disease.

The place suggested as most suitable for treatment was mainly the family home $(80.9 \%) ; 12.8 \%$ underlined that hospitals are not appropriate places for care of PWE (Table 4).

\subsection{Attitudes toward people with epilepsy}

Some restrictions were highlighted especially with respect to activities, relationships with other community members, and travel (Table 5). Thus, $44.7 \%$ reported that PWE should not travel alone and $21.3 \%$ said they should not perform heavy work. Nearly half of the respondents (46.8\%) reported that PWE should not be in contact with children, and 31.9\% said they should not even approach pregnant women.

With respect to forbidden places, $42.6 \%$ of respondents reported that PWE should avoid distant cities and 19.1\% claimed they should also avoid nearby communities. Moreover, $14.9 \%$ said they should not join local celebrations. It was also noted that the Guaraní believe that PWE should avoid some specific behaviors such as being involved in problems (23.4\%) and drinking alcohol (Table 5). These actions are in fact considered by the interviewees triggering factors that could worsen the course of the disease.

\subsubsection{The biomedical system from Guarani perspective}

Fifty-one percent of respondents reported that the biomedical approach (use of AEDs) could be effective in controlling epilepsy, whereas $21.0 \%$ appeared not to be confident in this approach. More than a quarter of subjects (25.5\%) said that Karaï (white doctors) do not provide proper explanations, and $21.3 \%$ considered them too cold and detatched (Table 6). Conversely, local nurses seemed to be well accepted by the community, as $62.0 \%$ of respondents declared

Table 3

Those able to manage PWE according to the Guaraní.

\begin{tabular}{lc}
\hline Answer & $N(\%)$ \\
\hline For people belonging to folk sector & \\
Family & $42(89.3)$ \\
Householder members & $2(4.3)$ \\
Community members & $2(4.3)$ \\
No answer & $1(2.1)$ \\
For people belonging to traditional sector & \\
Shamans' assistants, Poro-Peyu Vae & $16(34.0)$ \\
Every community member who knows a method & $11(23.4)$ \\
Shamans, El Paye & $9(19.1)$ \\
Biomedical doctors, Karai & $6(12.8)$ \\
Natural medicine practitioners & $5(10.6)$ \\
$\quad$ Total & $47(100.0)$
\end{tabular}


Table 4

Health care facilities for the treatment of PWE from the Guaraní perspective.

\begin{tabular}{lc}
\hline Answer & $N(\%)$ \\
\hline Suitable places & $38(80.9)$ \\
Home & $4(8.5)$ \\
Natural medicine practitioner's house & $3(6.4)$ \\
Hospital & $1(2.1)$ \\
Community & $1(2.1)$ \\
No answer & \\
Unsuitable places & $20(42.6)$ \\
Out of home & $10(21.3)$ \\
Empty house & $6(12.8)$ \\
Hospital & $4(8.5)$ \\
Far cities & $6(12.8)$ \\
Others & $1(2.1)$ \\
No answer & $47(100.0)$ \\
Total &
\end{tabular}

they were satisfied with their work and only $26.0 \%$ considered them unqualified. Despite this, a vast majority of people (83.0\%) appeared to favor the possibility of a combination of traditional and biomedical medicine. However, some of the respondents who disagreed with this collaboration specified that before taking any AEDs, PWE should consult a traditional medicine practitioner: "You should contact the Karai when our remedies cannot cure the disease"; "People who seek Karai attention are those that have already been to the Paye without having any benefit."

\subsection{Point of view of health care personnel}

\subsubsection{Characteristics of the interviewed}

Five people in the medical field were interviewed (two women and three men). All were nurses working in different Guaraní

Table 5

Guaraní attitudes toward epilepsy.

\begin{tabular}{lc}
\hline Answer & $N(\%)$ \\
\hline Forbidden activities & \\
Traveling alone & $21(44.7)$ \\
Heavy works & $10(21.3)$ \\
Alcohol drinking & $6(12.8)$ \\
Walking at night & $3(6.4)$ \\
Farm works & $2(4.3)$ \\
Going far away & $1(2.1)$ \\
Other & $2(4.3)$ \\
No answer & $2(4.3)$ \\
Forbidden contacts & \\
Children & $22(46.8)$ \\
Pregnant women & $15(31.9)$ \\
Religious leaders & $1(2.1)$ \\
Other & $1(2.1)$ \\
No answer & $8(17.0)$ \\
Forbidden places & \\
Distant cities & $20(42.6)$ \\
Neighboring communities & $9(19.1)$ \\
Celebrations & $7(14.9)$ \\
Anywhere outside the home & $6(12.8)$ \\
Farms & $1(2.1)$ \\
Other & $1(2.1)$ \\
No answer & $3(6.4)$ \\
Nonrecommended behaviors & $12(25.5)$ \\
Being involved in problems & $47(100.0)$ \\
Drinking alcohol & $7(23.4)$ \\
Losing self-esteem & $5(10.9)$ \\
Discontinuing treatment & $3(6.4)$ \\
Apathy & $1(2.1)$ \\
Blaming for the disease & $1(2.1)$ \\
Being nervous & $1(2.1)$ \\
Sexual activity & $1(2.1)$ \\
Other & $5(10.6)$ \\
No answer & \\
Total & \\
\hline
\end{tabular}

Table 6

Opinions about doctors offered by the Guaraní.

\begin{tabular}{lc}
\hline Answer & $N(\%)$ \\
\hline Unable to provide explanations & $12(25.5)$ \\
Detached & $10(21.3)$ \\
Impolite & $3(6.4)$ \\
Arrogant & $2(4.3)$ \\
Not confident in our competence & $1(2.1)$ \\
Disrespectful & $1(2.1)$ \\
Despise traditional methods & $3(6.4)$ \\
Do not follow-up patients & $1(2.1)$ \\
Give wrong treatments & $6(12.8)$ \\
No answer & $8(17.0)$ \\
$\quad$ Total & $47(100.0)$ \\
\hline
\end{tabular}

communities. Four of them had been working in these communities more than 5 years and were fluent in the local language.

\subsubsection{Causes of epilepsy}

Questioned about the origin and causes of epilepsy, two nurses pointed out environmental agents as causal factors, whereas the others indicated eating habits and lack of participation in community life and traditions.

\subsubsection{The relationship between health care providers and patients}

Concerning factors that could represent a barrier to adherence to AED treatment, two nurses stated that the people probably do not trust the biomedical sector, whereas the other nurses attributed this to the negative influence of traditional healers or to the difficulty faced by medical staff in being understood by the communities.

When asked "Which are the biggest obstacles in your relationship with patients?" the nurses suggested the discomfort that people feel during the medical examination, the low level of education of individuals, a strong belief in traditional medicine, and little confidence in the biomedical approach. Lack of family support was also considered an obstacle to this relationship.

When asked to propose a strategy to improve the quality of the relationship with patients, the majority suggested strengthening ties of friendship with all community members to show respect for the culture and for the role of traditional healers and medicine.

\section{Discussion}

This study represents a starting point for understanding Guaraní sociocultural beliefs about epilepsy, a topic about which little is known because of the small number of research studies carried out to date. This evaluation was realized in the attempt to gather useful information on which to base an intervention program in a region where the lifetime prevalence of epilepsy has been estimated to be between 12.3 and 15.3/1000 [21] and where untreated epilepsy represents a critical public health issue with a very high treatment gap.

The research benefited from a multidisciplinary and cross-cultural approach supported by the Centre for Anthropological Research of the Teko Guarani (an institution founded in 1989 with the aim of restoring and enhancing Guaraní culture), which provided a team of local investigators very familiar with Guaraní culture and able to speak Guaraní fluently.

The screening of participants, performed directly within the communities, permitted establishment of a sample with good age and gender ranges and identification of key figures like community elders, considered the holders of tradition and culture. The participation of local health care personnel was also considered very important in this investigation because of their role as intermediates between the communities and the biomedical sector.

The two-phase methodology conducted through FGDs and interviews permitted us not only to obtain qualitative information, but 
also to make quantitative estimations. Despite the small sample size and the reduced number of communities selected, we believe our results to be representative of the global view and beliefs of the Guaraní. In addition to Guaraní perceptions of the disease, interpretation of the term used to describe epilepsy seems to be the key to understanding attitudes. In fact, the expression mano-mano is meaningful. It refers to the idea of a round trip between life and death. This concept addresses the loss of consciousness and shows that epilepsy is recognized mostly in terms of generalized seizures. The uncertain state between life and death is seen as a kind of "third possible condition" for a human being, a state that generates hesitation over what attitude to hold. PWE are omano-mano-vae, the "undeads," different from the other members of the community and considered both as victims of this life-death relationship and as enablers of the meeting of these two worlds.

Regarding the causes, the importance of human action in triggering the disease is evident. We could differentiate objective actions such as food intake, alcohol consumption, and heredity and more subjective actions, concerning mainly cultural aspects, such as the violation of certain rules established by the community. A common denominator that linked these two classes of causes is fasting. The lack of respect for traditions is reflected by the failure to observe the Yekuaku and by the consumption of foods that lead to the appearance of the disease. In a larger vision, the infringement committed by the individual acquires a broader dimension: it is related to a failure of the entire community, whose primary role is to enforce traditions. If a community member has committed an error, then the community must have previously made a mistake. This vision moves the idea of fault from an individual to the entire community and thus avoids isolation and condemnation of the individual.

The concept of epilepsy as an embodied disease with more natural than supernatural causes expressed by the Guaraní appears to differ from those documented in other traditional societies like some African populations, which consider epilepsy a curse, an incarnation of evil spirits, a punishment for a sin, or a contagious disease [22-24]. It also differs from the view of some Asian populations that have attributed epilepsy to mental illness or trauma that causes a weakening of the nervous system $[25,26]$. The Guaraní vision deviates as well from the view of other native tribes of Central and South America that regard the disease as supernatural, mainly due to witchcraft and strictly associated with religious beliefs [27].

From the point of view of health care personnel, the causes of epilepsy reflect in part the views of the communities, as they referred commonly to environmental factors, eating habits, and detachment from traditions.

Regarding treatment of epilepsy, for Guaraní, the community seems to play a major role. If the outbreak of the disease is related to the failure to observe traditions that represent the foundations of the community, it must be the same culture that proposes solutions. The most appropriate people able to cure the disease are identified as shamans' assistants, family members, and community members. Moreover, women seem to represent the most important figures, within the family, for providing care to PWE.

The place of care is also strictly linked to the community and represented by home, the place where the family lives. The reluctance to use therapeutic solutions from other cultures and the vision of the hospital as an inappropriate site for care are direct consequences: these places are not part of Guaraní culture.

The natural origin of epilepsy is another well-stuctured aspect of Guaraní views on this disease. The preference for shamans' assistants instead of shamans is in fact justified by the belief that shamans are involved in the treatment of diseases with mainly supernatural, spiritual, or magical causes, whereas their assistants deal with disease of natural origin. Shamans are, conversely, the principal health care providers required to treat PWE in other Native American cultures characterized by a more magic-religious concept [27]. The link with nature is also pointed out in terms of treatments: drinking herbal infusions is not sufficient; it is also important to take into account natural phenomena such as the lunar cycle or the day/night transition.

The representation of epilepsy as a state of human being and the perception of this in a vision that involves the entire community allow an interpretation of Guaraní attitudes toward PWE. Guaraní PWE are rarely condemned, misjudged, or isolated as in other cultures. Apparently, PWE do not represent a threat to the Guaraní, who seem to hold the attitude of helping and protecting PWE. As noted, the restrictions and prohibitions cited by the Guaraní appear to derive from the need to take care of PWE, as heavy work, traveling alone, and being involved in problems are believed to worsen the condition or trigger seizures in PWE. However, the advice to avoid contact between PWE and children or pregnant women raises some questions that could be related to a belief that was not mentioned: possible transmission of the disease to those who are considered the weakest and most defenseless in the community.

Despite the tendency to turn to more traditional treatment, people from these communities appear to favor a combination of biomedical and traditional care systems. Furthermore, we noted a pragmatism in the assertion by some that the biomedical sector should be approached when traditional medicine does not provide an adequate solution.

The local health care personnel might assume a very important role as a link between traditional and biomedical sectors. Local nurses are very well accepted within the population; they share their views and their culture and speak an understandable and reliable language. Conversely, biomedical doctors are considered very distant from Guaraní culture and unable to provide explanations. Specific programs aimed at improving communities' knowledge and understanding of the biomedical field are needed.

Our findings show that the Guaraní view of epilepsy seems to be overt and favorable enough to a biomedical approach. Furthermore, this study clearly highlights that, to ensure the success of future programs using AEDs, a multidisciplinary program is needed that provides education and information to PWE and the community, involves local health care providers, and recognizes the role of traditional medicine in the therapeutic process.

\section{Ethical approval}

We confirm that we have read the Journal's position on issues involved in ethical publication and affirm that this report is consistent with those guidelines.

\section{Conflict of interest statement}

We certify that none of the authors had any conflict of interest to disclose.

\section{Acknowledgments}

We are grateful to Father Tarcisio Dino Ciabatti, coordinator of the agreement between the Ministerio de Salud y Deportes and the Vicariato Apostólico de Camiri, for his encouragement and cooperation; to the field team members, for their valuable assistance during the survey; and to the members of the community of Cañon de Segura and Rodeo; for their cooperation and willingness to take part in the study. We thank the local health authorities and the Asamblea del Pueblo Guaraní for their encouragement and cooperation. We also thank Professor CatherineMarie Dubreil whose comments on this article were invaluable.

This work was supported by a grant from the Italian Ministry of Foreign Affairs-Development Cooperation within the project Strengthening of Health Services in the Bolivian Chaco: A Community Based Perspective (8759/UCODEP/BOL) and by the European Commission within the 7th Framework Programme, under the COHEMI project (COordinating resources to assess and improve HEalth status of MIgrants from Latin America) grant agreement no. FP7-GA-261495. 


\section{References}

[1] Kale R. Global Campaign against Epilepsy: the treatment gap. Epilepsia 2002;43 (Suppl 6):31-3.

[2] Diop AG, De Boer HM, Mandlhate C, Prilipko L, Meinardi H. The global campaign against epilepsy in Africa. Acta Trop 2003;87:149-59.

[3] Begley CE, Baker GA, Beghi E, et al. Cross-country measures for monitoring epilepsy care. Epilepsia 2007;48:990-1001.

[4] Meyer AC, Dua T, Ma J, Saxena S, Birbeck G. Global disparities in the epilepsy treatment gap: a systematic review. Bull WHO 2010;88:260-6.

[5] Meinardi H, Scott RA, Reis R, Sander JW. The treatment gap in epilepsy: the current situation and ways forward. Epilepsia 2001;42:136-49.

[6] De Boer HM, Mula M, Sander JW. The global burden and stigma of epilepsy. Epilepsy Behav 2008:12:540-6.

[7] Kwan P, Brodie M. Phenobarbital for the treatment of epilepsy in the 21st century: a critical review. Epilepsia 2004;45:1141-9.

[8] Wang WZ, Wu J, Ma G, et al. Efficacy assessment of phenobarbital in epilepsy: a large community-based intervention trial in rural China. Lancet Neurol 2006;5: 46-52.

[9] Baskind R, Birbeck GL. Epilepsy-associated stigma in sub-Saharan Africa: the socia landscape of a disease. Epilepsy Behav 2005;7:68-73.

[10] Chisholm D, for WHO-CHOICE. Cost-effectiveness of first-line antiepileptic drug treatments in the developing world: a population-level analysis. Epilepsia 2005;46:751-9.

[11] Nicoletti A, Reggio A, Bartoloni A, et al. A neuro-epidemiological survey in rural Bolivia: background and methods. Neuroepidemiology 1998;17: 273-80.

[12] Nicoletti A, Reggio A, Bartoloni A, et al. Prevalence of epilepsy in rural Bolivia: a door-to-door survey. Neurology 1999;53:2064-9.

[13] Nicoletti A, Sofia V, Vitale G, et al. Natural history and mortality of chronic epilepsy in an untreated population of rural Bolivia: a follow-up after 10 years. Epilepsia 2009;50:2199-206.
[14] Carod FJ, Vazquez-Cabrera C. A transcultural view of neurological and mental pathology in a Tzeltal Maya community of the Altos Chiapas. Rev Neurol 1996;24:848-54.

[15] Carod-Artal FJ, Vazquez-Cabrera C. Neurological anthropology among the Kamayura Indians of the alto Xing u. Rev Neurol 2001;32:688-95.

[16] Carod-Artal FJ, Vazquez-Cabrera CB. Ethnographic study of neurological and mental diseases among the Uru-Chipaya peoples of the Andean Altiplano. Rev Neurol 2005;41:115-25.

[17] Carod-Artal FJ, Vazquez-Cabrera CB. Mescaline and the San Pedro cactus ritual: archaeological and ethnographic evidence in northern Peru. Rev Neurol 2006;42: 489-98.

[18] El Sharkawy GE, Newton C, Hartley S. Attitudes and practices of families and health care personnel toward children with epilepsy in Kilifi, Kenya. Epilepsy Behav 2006;8:201-12.

[19] De Boer HM. Out of the Shadows: a global campaign against epilepsy. Epilepsia 2002:43(Suppl 6):7-8.

[20] Instituto Nacional de Estadıstica, INE. Indicadores Sociodemograficos por Provincias, Censo; 1992. La Paz, Bolivia.

[21] Burneo JG, Tellez-Zenteno J, Wiebe S. Understanding the burden of epilepsy in Latin America: a systematic review of its prevalence and incidence. Epilepsy Res 2005;66:63-74.

[22] Newton CRJC, Gero B. The epilepsies among rural blacks. South Afr Med J 1984;66: 21-3.

[23] Whyte SR. Constructing epilepsy: images and context in East Africa. In: Ingstad B, Whyte SR, editors. Disability and culture. Berkeley: Univ. of California Press; 1995;184-233.

[24] Pickering C. How to help with epilepsy. Nairobi: Kenya Association for the Welfare of Epileptics; 2002.

[25] Nguyen A. Cultural and social attitudes towards mental illness in Ho Chi Minh City, Vietnam. Stanford Univ Res J 2003;2:27-31.

[26] Jacoby A, Wang W, Dang Vu T, et al. Meanings of epilepsy in its sociocultural context and implications for stigma: findings from ethnographic studies in local communities in China and Vietnam. Epilepsy Behav 2008;12:286-97.

[27] Carod-Artal FJ, Vasquez-Cabrera CB. An anthropological study about epilepsy in native tribes from Central and South America. Epilepsia 2007;48:886-93. 